\title{
An Empirical Study for Dynamic TIPP Policy Using XCS with Knowledge Rules
}

\author{
Mei-Chih Chen ${ }^{1,2}$ Ming-Chia Huang ${ }^{1} \quad$ An-Pin Chen ${ }^{1}$ \\ ${ }^{1}$ Institute of Information Management, National Chiao Tung University, \\ ${ }^{2}$ Department of Information Management, Ming Hsin University of Science and Technology
}

\begin{abstract}
The purpose of this empirical study is intended to investigate XCS (Extended Classifier System) based model with knowledge rules for dynamic TIPP (Time Invariant Portfolio Protection) policy. There are two XCS-based agents in the proposed model (MA-TIPP).One agent dynamically optimizes Multiple and Tolerance variables which are concerned as the important parameters of TIPP and recommend trading. The other one is aimed to use $80 \%$ accuracy historical rules retained by classifier system to improve the previous agent prediction accuracy. The Multiple and Toleranc parameters which are optimized by GA and stock technical indexes such as Moving Average(MA), Moving Average Convergence and Divergence (MACD), Stochastic Line(KD), Relative Strength Index (RSI), Close and Volume are used as the input factors of classifier system. This proposed model is evaluated by $80 \%$ insurance and periods of TAIEX (Taiwan weighted) from 1996 to 2004. The experimental results are also compared with single XCS agent model (SA-TIPP) without using historical knowledge rules.
\end{abstract}

Keywords: Multi-Agent System, Time Invariant Portfolio Protection, Extended Classifier System

\section{Introduction}

An investor faces the investment risk including the system risk and non-system risk. The descreasing of non-system risk might diversify portfolio into different commodities, so the investor can avoid the individual factor to get more risks. The portfolio insurance concept is paid some fee to get free from system risk or losing when the market is falling dramatically. But investors also can make a profit when market is rising.

CPPI (Constant Portion Portfolio Insurance) and TIPP are the common methods of portolio insurance strategy. TIPP [4] is aimed to modify CPPI strategy using fixed Floor. For instance, the initial asset worths $\$ 1000$ and start with $80 \%$ Floor percentage. TIPP policy Floor never decline below $\$ 800$ but it might raise up. This policy depends on investor's favorite. If the Multiple is too big, the assets might not be protected. If it's too small, the marketing percentage will become too low to get the expected profit. TIPP policy is time sensitive, its performance is relative with the starting time [8].

Our research bases on the Extended Classifier System to construct MA-TIPP strategy model. TAIEX is the study objective. The purpose of this research is to enhance XCS prediction accuracy. Eventually, Sharpe ratio and accumulated return ratio are the criteria to evaluate the proposed MA-TIPP and SA-TIPP model.

\section{Literal Review}

CPPI strategy [1] simple parameter is set up for the fixed Floor purpose. The Floor percentage is unchanged, but the Floor varies with market in the insurance period. Trade and rebalance are executed when active assets vary excessing Tolerance.

TIPP strategy[4] is a modified version of CPPI. The difference between CPPI and TIPP is to choose the bigger one between the previous Floor and the Floor percentage of current portforlio value. The new Floor always keeps gains and protects the Floor absolutely.

Dynamic portfolio insurance strategy should adjust contiously to get the expected result [5], In bullish trend, it loses the premium [6]. In bearish market, it could protect active assest from losing. When the market is fluctuating, transaction cost will affect the return [3]. Technical analysis is not a way to accurate the stock price, but it really help the successing probability [12]. Such as CRISMA system [10] use Cumulative Volume, Relative Strength Index, Moving Average to do buy and sell decision. With transaction cost or not, CRISMA outperformed the Buy\&Hold strategy. Gencay and Stengos adopt Price and Volume Moving Averages, investigate Dow Jones index, expose Volume can improve predicting [7]. Mark 
uses 9K, 9KD, 18ADX, 18MACD, and S\&P500 etc. as neural network input factors, this model can predict well [9]. Our reserach adopts above metioned technical index describing market states as XCS input factors.

Classifier System based on Holland proposed Cognitive System. Butz and Wilson modify Classifier System to improve its effective, is called Extended Classifier System [2]. It suits on dynamic environment predictor work. Recently, Classifier System is applied in financial market. Such as investing IBM stock, the gain of Classifier System is better than Buy\&Hold strategy [11]. In foreign exchange, Classifier Sysetm also generates dynamic rules predicting result. Classifier System has been applied in wide variety of domain such as design, medicine, classification, control and others.

\section{The Design of MA-TIPP Model}

This model of TIPP combines GA and XCS, Genetic Algorithm (GA) initializing Multiple, Tolerance parameters and then input to XCS to predict market trend for trade and rebalance operating and adjust Multiple, Tolerance to market state, See fig.1. There are two XCS-based agents in the proposed model (MA-TIPP). Predict-XCS dynamically optimizes Multiple and Tolerance variables which are concerned as the important parameters of TIPP and recommend trading. $\mathrm{KR}-\mathrm{XCS}$ is aimed to use $80 \%$ accuracy historical rules retained by classifier system to improve the Predict-XCS prediction accuracy.

We adopt MA, KD, MACD, RSI, Close, Volume being indicator of market states. After preprocessed, those data as Predict-XCS and KR-XCS input condition factors. Each XCS parameter setting is referenced from Butzs setting [2]. The rule population is extended to 3000 in order to accomadate complex rules. The training period is 5 years and testing period is 3 years. The initial Multiple and Tolerance are optimized by GA, then through XCS's evolution to produce adequate Multiple, Tolerance and market rising/falling prediction. SA-TIPP is according to the Predict-XCS output to operate trading and rebalancing. See Figure 2. The MA-TIPP model is according to the Predict-XCS and KR-XCS prediction to operate trading and rebalancing. If Predict-XCS and KR-XCS have the same prediction, MA-TIPP do trade and rebalance, otherwise do nothing.

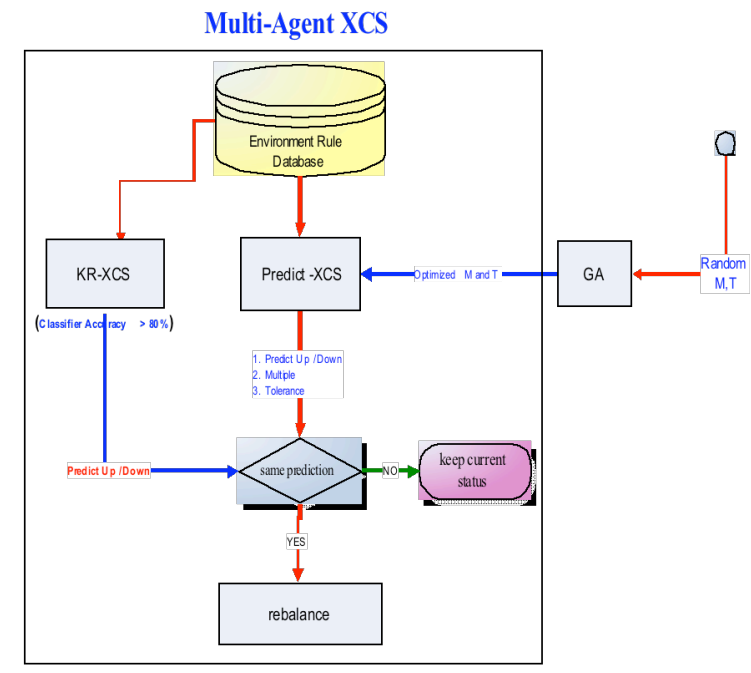

Fig.1: MA-TIPP Model

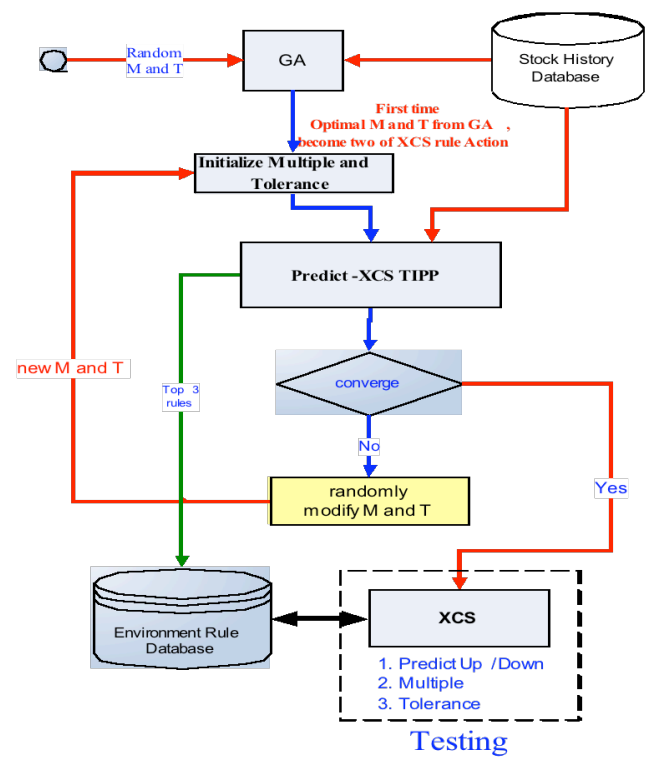

Fig.2: Predict XCS Model (SA-TIPP)

Following are going to explain classifier design and how to generate initial rules database.

Each classifier (rule) consists of condition and action part, see table 1 . The condiction part is the environment states detected by classifier system, and the action part is the recommendation of classifier system.

Table 1: Content of Classifier

\begin{tabular}{|l|l|}
\hline \multicolumn{1}{|c|}{ Condition part } & \multicolumn{1}{c|}{ Action part } \\
\hline $\begin{array}{l}\text { assembled by state of } \\
\text { technical index, Close, } \\
\text { Volume }\end{array}$ & $\begin{array}{l}\text { Market } \\
\text { rising/falling } \\
\text { prediction, } \\
\text { Multiple, Tolerance }\end{array}$ \\
\hline
\end{tabular}




\subsection{Condition Part of Classifier}

Classifier's condition part is converted by state rules shown in table 2 . Each classifier has 9 bits representing market condition.

Table 2 State Rules

\begin{tabular}{|c|c|c|}
\hline $\begin{array}{l}\text { Technical } \\
\text { index }\end{array}$ & condition & $\begin{array}{c}\text { bit } \\
\text { value }\end{array}$ \\
\hline \multirow{2}{*}{ 5MA Close } & Close $>5 \mathrm{MA}$ & 1 \\
\hline & Close $<=5 \mathrm{MA}$ & 0 \\
\hline \multirow{2}{*}{ 22MA Close } & Close $>22 \mathrm{MA}$ & 1 \\
\hline & Close $<=22 \mathrm{MA}$ & 0 \\
\hline \multirow{2}{*}{$5 \quad 22 \mathrm{MA}$} & $5 \mathrm{MA}>22 \mathrm{MA}$ & 1 \\
\hline & $5 \mathrm{MA}<=22 \mathrm{MA}$ & 0 \\
\hline \multirow{2}{*}{$560 \mathrm{MA}$} & $5 \mathrm{MA}>60 \mathrm{MA}$ & 1 \\
\hline & $5 \mathrm{MA}<=60 \mathrm{MA}$ & 0 \\
\hline \multirow{2}{*}{ 9KD } & $9 \mathrm{~K}>9 \mathrm{D}$ & 1 \\
\hline & $9 \mathrm{~K}<=9 \mathrm{D}$ & 0 \\
\hline \multirow{2}{*}{$5 \quad 22 \mathrm{RSI}$} & $5 \mathrm{RSI}>22 \mathrm{RSI}$ & 1 \\
\hline & $5 \mathrm{RSI}<=22 \mathrm{RSI}$ & 0 \\
\hline \multirow{2}{*}{ 9MACD } & $\mathrm{DIF}>\mathrm{MACD}$ & 1 \\
\hline & $\mathrm{DIF}<=\mathrm{MACD}$ & 0 \\
\hline \multirow{4}{*}{ Volume,Close } & $\begin{array}{l}\text { (Close>prev. Close) } \\
\text { and } \\
\text { (Volume }>\text { prev. } \\
\text { Volume) }\end{array}$ & 11 \\
\hline & $\begin{array}{l}\text { (Close }<=\text { prev. } \\
\text { Close) and } \\
\text { (Volume }<\text { prev. } \\
\text { Volume) }\end{array}$ & 10 \\
\hline & $\begin{array}{l}\text { (Close }>\text { prev. } \\
\text { Close) and } \\
\text { (Volume }<\text { prev. } \\
\text { Volume) }\end{array}$ & 01 \\
\hline & $\begin{array}{l}\text { (Close }<\text { prev. } \\
\text { Close) and } \\
\text { (Volume }>\text { prev. } \\
\text { Volume) }\end{array}$ & 00 \\
\hline
\end{tabular}

\subsection{Action Part of Classifier}

The action part has 3 items which are market rising/falling prediction, Multiple, and Tolerance. One bit represents market prediction, two bits for Multiple, and the same as Tolerance. If the action part value is 15235, it means market as being bullish and Multiple, Tolerance has better being set to $5.2,3.5 \%$ respectively.

\subsection{Initialization of Rule Database}

This study adopts 5MA, 22MA, 60MA, 9KD, 9MACD, 5RSI, 22RSI, Close, Volume describing stock market states. The initial rules are generated by training steps until the result converged, or randomly add value $[-1.0,1.0]$ to Multiple, and Tolerance and then choosing top 3 rules into a rule database. If rule of accuracy is same, the rule is chosed by roulette wheel selection.

\section{Experiment and Result}

\subsection{Data source}

The experimental Taiwan weighted data download from Yahoo! Finance website[13]. The data are from $1991 / 01 / 03$ to $2005 / 01 / 03$. The initial capital is NT\$1 billions. Transaction cost is NT\$150 and tax is $0.25 \%$ and risk free fixed deposit ratio is $1.31 \%$.

\subsection{Relative design of GA}

The initial Multiple and Tolerance of Predict-XCS are generated by GA. The relative design is metioned below.

Chromsome consists of gene Multiple and gene Tolerance.

$$
\begin{aligned}
& \text { Multiple } \\
& \mathrm{M} \subset\{00,11,12,13 \sim 97,98,99, \mathrm{~A} 0\} \\
& \text { Tolerance } \\
& \mathrm{T} \subset\{000,101,102, \sim 998,999, \mathrm{~A} 00\}
\end{aligned}
$$

Population size : 500

Crossover rate : 0.5 (two-point crossover)

Mutation rate : 0.005

Fitness value : Sharpe Ratio

Termination condition : difference between offspring and parent $<0.00001$

Traing period is 5 years.

\subsection{Experimental result}

The table 3 shows MA-TIPP and SA-TIPP experimental result with Sharpe ratio and accumulated return ratio. 
table 3 : MA-TIPP \& SA-TIPP Performance

Comparative

\begin{tabular}{|l|l|l|r|l|}
\hline $80 \%$ Floor & \multicolumn{2}{|l|}{ MA-TIPP } & \multicolumn{2}{l|}{ SA-TIPP } \\
\hline Period & $\begin{array}{l}\text { Sharpe } \\
\text { Ratio }\end{array}$ & $\begin{array}{l}\text { Return } \\
\text { Ratio }\end{array}$ & $\begin{array}{l}\text { Sharpe } \\
\text { Ratio }\end{array}$ & $\begin{array}{l}\text { Return } \\
\text { Ratio }\end{array}$ \\
\hline $1996-1998$ & 1.99 & $106.06 \%$ & 2.00 & $106.57 \%$ \\
\hline $1997-1999$ & 1.96 & $109.61 \%$ & 2.09 & $114.75 \%$ \\
\hline $1998-2000$ & 2.88 & $158.90 \%$ & 2.84 & $163.04 \%$ \\
\hline $1999-2001$ & 1.69 & $97.51 \%$ & 1.65 & $95.63 \%$ \\
\hline $2000-2002$ & 1.05 & $56.44 \%$ & 1.14 & $61.90 \%$ \\
\hline $2001-2003$ & -0.13 & $-3.60 \%$ & -0.19 & $-5.75 \%$ \\
\hline $2002-2004$ & -0.37 & $-13.97 \%$ & -0.39 & $-14.79 \%$ \\
\hline
\end{tabular}

The MA-TIPP performance doesn't outperform SA-TIPP policy. It is obvious that KR-XCS didn't improve XCS's prediction accuracy.

\section{Conclusion}

This empirical study is try to use historical $80 \%$ accuracy rules improving classifier system prediction accuracy, but it doesn't success. Classifier Systems are rule-based system. They are trying to find out the best rule through trial and error via the detective condition. This empirical study's result reveals extended classifier system always keeping good rules. Our future research will aim to different insurance period, diversity commodities, different training period and testing period to improve the XCS-based TIPP model.

\section{Reference}

[1] Fischer Black, Robert Jones, "Simplifying Portfolio Insurance", Journal of Portfolio Management, pp.48-51, 1987.

[2] M.V.Butz, , S.W. Wilson. "An Algorithmic Description of XCS", Soft Computing 6, pp.144-153, 2002.

[3] R.G. Clarke, R.D. Arnott, "The Cost of Portfolio Insurance: Tradeoffs and Choice”, Financial Analysts Journal, pp.35-47, 1987.

[4] T. Estep, M.Kritzman, "TIPP: Insurance without Complexity", Journal of Portfolio Management 14, summer, pp.38-42, 1988.

[5] E.S. Ethan, "Rebalance Disciplines for Portfolio Insurance", Journal of Portfolio Management, pp.59-62, 1986.

[6] C. B.Garcia, F. J.Gould,"A Note on the Measurement of Risk in a Portfolio", Financial Analysts Journal, pp.61-69, 1987.

[7] R.Gencay, T.Stengos, "Moving Average Rules, Volume and the Predictability of
Security Returns with Feedforward Networks" , Journal of Forcasting 17, pp.401-414, 1988 .

[8] S. Kenneth Choie, Eric J. Seff, "TIPP : Insurance without complexity: Comment", Journal of Portfolio Management, pp.107-108, 1989.

[9] Mark etc. "Using neural networks in market analysis", Technical Analysis of Stocks and Commodities , 9, pp.18-21, 1991.

[10] S.W.Pruit, R.E.White," The CRISMA Trading System: Who Says Technical Analysis Can't beat the market?", Journal of Portfolio Management Spring, pp.55-58, 1988.

[11] S.Schulenburg, P.Ross, "Explorations in LCS Models of Stock Trading", Advances in Learning Classifier Systems : 4th International Workshop, IWLCS 2001, San Francisco, CA, USA, July 7-8. Revised Papers ,pp.151, 2001.

[12] G.Soros, The Alchemy of Finance: reading the mind of the market, J.Wiley, 1994.

[13] http://finance.yahoo.com 